\title{
Inclusión de perros en la rehabilitación emocional en casos de violencia contra las mujeres
}

\author{
Inclusion of dogs in emotional rehabilitation in cases \\ of violence against women
}

\author{
Viviana CASTEllanos-SuÁREZ \\ Universidad Juárez Autónoma de Tabasco \\ Departamento de Psicología.
}

ORCID: https://orcid.org/0000-0003-2506-8207

Recibido: $17 / 09 / 2019$

Aceptado: 17/10/2019

doi: https://doi.org/10.20318/femeris.2020.5155

Resumen. Ante la violencia contra las mujeres existen numerosos modelos de prevención y atención psicoeducativos, siendo escasos los de intervención terciaria concretamente de rehabilitación de los daños emocionales que dejo la violencia en las mujeres.

En este estudio se pretende dar a conocer el relato de seis mujeres que han vivido violencia y que reportan tener un animal de compañía en este caso un perro y con ello identificar los beneficios que han tenido en su recuperación emocional y en su rehabilitación.

Se aprecia cómo los animales apoyan a las mujeres en establecer y manejar vínculos positivos, a sortear encrucijadas emocionales y recuperar el control de emociones y en ser catalizadores para restaurar la fuerza y la energía. Como área de oportunidad se requiere elaborar protocolos específicos para poder emigrar de una inclusión animal a una Terapia Asistida por animales en casos de violencia contra las mujeres.

Palabras clave: Inclusión terapéutica con perros, rehabilitación emocional, violencia contra las mujeres.

Abstract. In the face of violence against women, there are numerous models of prevention and psychoeducational care, with few tertiary intervention specifically rehabilitation of emotional damage left by violence in women. This study aims to publicize the story of six women who have experienced violence and who report having a companion animal in this case a dog and thereby identify the benefits they have had in their emotional recovery and rehabilitation. It is appreciated how animals support women in establishing and managing positive links, overcoming emotional crossroads and regaining control of emotions and being catalysts to restore strength and energy. As an area of opportunity it is necessary to develop specific protocols to be able to migrate from an animal inclusion to an Animal Assisted Therapy in cases of violence against women. women.

Keywords: Therapeutic inclusion with dogs, emotional rehabilitation, violence against

*viviana.castellanos@ujat.mx/vivihermosa_70@hotmail.com 


\section{Introducción}

La violencia contra las mujeres es reconocida en términos de pandemia, se registra que una de cada tres mujeres ha soportado violencia física o sexual principalmente por un compañero sentimental (ONU Mujeres, 2018). Estar calificada como pandemia y como una problemática de salud pública no es un tema reciente, desde 1995 en la Plataforma de Acción, aprobada por la Conferencia de Beijing, también se calificaba los mismos términos por sus características tanto en las cifras desbordantes como por abarcar todos los países del mundo.

Los daños y las consecuencias reclaman acciones concretas y efectivas de prevención, atención y rehabilitación, encaminados a erradicar la violencia contra las mujeres, incluso se ha considerado seriamente para su erradicación en los cinco objetivos clave para el 2030 de manera explicita el punto cuatro se refiere a eliminar todas las formas de violencia (ONU, 2019).

Las cifras de la violencia contra las mujeres refieren la prevalencia mundial a razón de una de cada tres de mujeres han sufrido violencia física y/o sexual (OMS, 2017), en el ámbito nacional, el caso de México se afirma que al menos 6 de cada 10 mujeres han vivido un incidente de violencia en su vida (ONU MUJERES México, 2017) sin embargo la cifra es creciente ya que se distinguen países donde hay un incremento de la violencia a 7 de cada 10 mujeres y se ha señalado que México es uno de ellos (Organización Mundial de la Salud, 2013).

El daño es tan grave que se ha llegado a equiparar la violencia contra las mujeres con la tortura por las repercusiones a largo plazo de los malos tratos reiterados que son física y psicológicamente devastadores y cuyas consecuencias abarcan el trauma psicológico, lesiones, embarazos no deseados, infertilidad y enfermedades que ponen en peligro la vida (Amnistía Internacional, 2001).

Ha sido identificada como una forma de tortura ya que se configuran tres elementos: el sufrimiento severo, físico y mental, la acción u omisión del estado ante los actos y la intencionalidad del acto y la motivación o el fin del acto para conseguir algo, se considera que la violencia contra las mujeres como una forma de tortura no es una aberración jurídica sino una interpretación más acorde con los estándares establecidos por tribunales internacionales de derechos humanos que permitan reforzar la prevención, pero sobre todo la reparación del daño y su eliminación (Rivero, 2014).

Es menester considerar también los efectos y consecuencias sobre la salud las cuales son claramente definidas en términos de inmediatas y agudas, duraderas y crónicas o mortales, identificando que a mayor maltrato, mayores repercusiones sobre la salud física y mental de las mujeres, se agrega que la violencia puede persistir mucho tiempo después de que haya cesado el maltrato y que las consecuencias se agravan cuando se sufre mas de un tipo de violencia o episodios repetidos con el transcurso del tiempo, clasificándose las consecuencias en cuatro áreas frecuentes, física, sexual y reproductiva, mental y conductual (Organización Mundial de la Salud, 2013).

Así mismo, las consecuencias y los daños de la violencia contra las mujeres se catalogan en términos de resultados mortales inmediatos como lo es el homicidio y el suicidio y no mortales, aunque potencialmente mortales (García, 2003). Al respecto el Informe 
Mundial sobre la violencia y la salud de la Organización Panamericana de la Salud (Krug, et al., 2003) logra diferenciar mas de cincuenta daños, enlistándolos como efectos físicos, sexuales y reproductivos, psíquicos y del comportamiento, así como de consecuencias mortales como la mortalidad materna, el suicidio y el homicidio.

Respecto a las secuelas cognitivas del daño, las afectaciones se pueden producir a través de tres vías: daño directo como consecuencia de golpes en la cabeza; daño indirecto a través de las alteraciones cerebrales que producen las secuelas psicológicas, especialmente, el estrés postraumático y daño indirecto a través del efecto que el cortisol, segregado en situaciones de estrés crónico, produce en el cerebro (Hidalgo, et al., 2012). Es necesario mencionar que este cortisol no se segrega y se recaptura en una sola ocasión, su generación es constante ante la amenaza suspendida ya que la violencia no cede ante la sola ausencia del violentador, este incluso puede estar en la mente de la persona y causar la segregación ante el recuerdo, al respecto se señala que aun terminada la relación, las mujeres e incluso sus hijos, no están libres de los efectos colaterales y expansivos de la violencia ya que esta no solo sucede durante la convivencia, también tras la separación, incluyendo otro tipo de violencias que antes no habían hecho su aparición (Fernández y González, 2012).

En lo que se refiere a la valoración del daño a la integridad psíquica y física como unidad indisoluble de la persona, se considera que los daños más graves en la violencia contra las mujeres no han sido reconocidos jurídicamente como el ocasionado en huella neurológica que deja la violencia en el sistema nervioso, tanto a nivel del sistema cerebroespinal como en el sistema neurovegetativo, se señala que el daño psicológico en este tipo de violencia se caracteriza entre otros, por que el desequilibrio y disfunción es resultante del evento traumático en el que se presenta un vínculo particularmente importante del cual se recibe maltrato mientras que lo que se esperaba era protección, apoyo, cercanía afectiva. (Lin Ching, 2003)

Otros daños se han identificado en el plan y proyecto de vida por las repercusiones que incluyen lesiones a su integridad como personas, pérdida de la libertad, oportunidades y posibilidades y hasta de la vida, pero sobretodo hay un elemento extra que se forma en la violencia contra las mujeres el cual logra irlas debilitando y menguando en su capacidad de respuesta, defensa, acción, este elemento es el miedo, el cual se constituye como un elemento importante de control patriarcal (Lagarde ,1996).

Exponer los efectos físicos, psicológicos y sociales que viven las mujeres permite visibilizar y problematizar, atender y prevenir la violencia, sin embargo, estos esfuerzos no han sido suficientes ya el número de personas que la padecen no cede y los daños entran en el terreno de lo irreversible, la violencia causa severo sufrimiento ya que excede los umbrales de lo humano, ubicándose en el contexto de una relación de dominación, control, sometimiento, asimétrica, tóxica y destructiva, tomando al otro como objeto simbólico conocida como violencia simbólica al existir la posición de dominado y dominante (Bourdieu, 2012).

Al ser la violencia progresiva, sostenida y repetitiva vulnera la salud, se aprecia como el abusador erosiona poco a poco, un punto débil ya existente de la persona abusada y más tarde explota los puntos que el mismo ha creado a partir del abuso, degradando su propio sentido de valor introduciendo en ella la opinión despectiva, esto lo consigue a través de la humillación paulatina y progresiva (Lamoglia, 2009). 
Ante tales consecuencias es altamente difícil generar modelos precisos o estrategias de atención concretas ya que la violencia crece, muta y se cronifica, sin duda los modelos multidisciplinarios realizan por ello una gran aportación, así mismo, hay que tomar en cuenta que una de las áreas con mas daño resulta ser el área psicológica ya que en ella recaen los demás tipos de violencia, es decir, el daño es transversal, ya que si bien la violencia física deja marcas y también deja heridas o huellas psicológicas, el control y sometimiento económico o patrimonial también deja su huella, la violencia sexual sin duda deja estragos huellas, marcas y heridas psicológicas en la psique humana, es decir todos los tipos de violencia convergen en el daño psicológico a largo plazo, de larga duración, crónico y en ocasiones irreversible, cuyas manifestaciones mas visibles son altos índices de angustia, ansiedad, estrés y depresión de tal forma que las huellas psicológicas que deja la violencia se traducen en secuelas (Arce, 2014).

Tanto las huellas psíquicas, como las secuelas conforman el daño psíquico el cual tiene manifestaciones somáticas y conductuales por ello la importancia de la rehabilitación emocional y en sí psicosocial, este daño se configura en una alteración o modificación patológica del aparato psíquico como consecuencia de un trauma que sobrepasa y desborda toda posibilidad de elaboración verbal o simbólica, conlleva una modificación o alteración de la personalidad expresada a través de síntomas, inhibiciones, depresiones, bloqueos y termina comprometiendo la esfera psíquica del sujeto incidiendo en las manifestaciones afectivas, volitivo y el intelectivo a lo cual se le conoce como daño psíquico o daño psicológico (Sessarego,2003).

En este daño psicológico queda una afectación emocional ya que la violencia que vivieron trajo estados emocionales de los cuales resulta difícil salir, llamados por encrucijadas emocionales tales como el miedo, la tristeza, la rabia, alegría y los pares que le corresponden, la ansiedad, la depresión, la violencia, euforia (Bizkarra,2005).

¿Cómo afrontar los daños emocionales de la violencia?, ¿Cómo recuperarse de este daño psíquico?, ¿Cómo apuntalar que las mujeres que han vivido violencia se recuperen de este flagelo? Una de las formas de favorecer esta rehabilitación es a partir del apoyo y asistencia de un animal de compañía, en este caso concreto perros, este tipo de intervención ha sido realizada en otras realidades, problemáticas o discapacidades donde asisten animales en el tratamiento, tal es el caso del autismo, ansiedad excesiva, o estrés, hiperactividad, personas con déficit de atención, problemas de aprendizaje, lenguaje, problemas motrices, Síndrome de Down, Ancianos, personas y niños con hospitalización prolongada, personas con cáncer, psicosis, esquizofrenia, personas en situación de cárcel y otras formas de violencia, en donde se reconoce el amplio campo de posibilidades y beneficios a la calidad de vida a partir de la terapia asistida con animales, esta mejora es tanto para si mismo como para otros y se da a partir de la relación con los animales y por la capacidad reparatoria del vínculo humano-animal (Muñoz ,2017).

Los animales son potenciadores de procesos terapéuticos y se reconoce la importancia relación inter-especies para recuperar la salud en el humano y sobretodo el manejo ético con los animales que es un aspecto que no puede dejarse sin mención (Suárez, 2019), de la misma manera se ha constado que la simple presencia de mascotas en este 
caso perros en una muestra de mujeres que han vivido violencia favoreció en su recuperación, la presencia de su perro y les permitió restablecer contacto, cercanía, vínculo consigo mismas y con los demás, además de fungir el animal como un regulador de emociones y del estado de ánimo, hallazgo que se identificó por su función como un depurador de la violencia (Castellanos, 2017).

Se ha propuesto la terapia asistida con animales como una forma de prevención y atención de la violencia de género durante la adolescencia, al facilitar el contacto con el animal y favorecer el contacto no violento ni dañino, por el contrario se constata que acariciar un animal, hace que el organismo libere hormonas y se tiendan a reducir los niveles de estrés y relajación, favoreciendo la integración interpersonal, modificando los pensamientos y estimulando el diálogo, detectándose mejoras en la autoestima y autocontrol (Soriano, 2018), en casos de violación a adolescentes también se advierte que la interacción, exposición y cercanía con el perro llega a mitigar síntomas de depresión y estrés postraumático (Hamama, et. al., 2011).

Concretamente la terapia asistida con animales es benéfica para los sobrevivientes de violencia doméstica ya que la sola interacción ayuda a reducir los niveles de trauma, estrés y ansiedad, se constata que el vínculo humano animal reduce la soledad, la depresión, aumenta la sensación de autoestima, empatía, bienestar psicológico y el sentido del propósito, se destaca que el beneficio más ventajoso de la terapia asistida con animales sobre otras formas de modalidades terapéuticas es la reciprocidad que proporciona ya que brinda al paciente la oportunidad tan anhelada de dar amor y afecto, así como recibirlo, propiciando la recuperación (Flynn, 2018).

De manera específica la combinación humano-perro, ha sido durante siglos importante al ser los perros de gran ayuda en la regulación de los humanos por sus capacidades superiores de audición y olfato, es una potente alarma sensorial al grado de saberse a salvo o ponerse alerta, esta capacidad correguladora es un componente importante del trabajo terapéutico con animales, un segundo elemento regulador es la actividad somato sensorial repetitiva y rítmica tiene en nuestros sistemas de respuesta al estrés, el acariciar, peinar, montar y caminar con los animales proporciona ritmo, calma y regulación a un individuo desregulado y un tercer elemento en restablecer una relación de confianza en situaciones de resiliencia en la recuperación del trauma (Teseschi y Jenkins, 2019).

Con base en lo anteriormente expuesto, se plantea como objetivo de este trabajo, conocer el papel que juegan los animales, en este caso los perros de compañía o conocidos también como mascotas, en la recuperación emocional en casos de violencia contra las mujeres y con ello determinar fortalezas y oportunidades. Para ello se obtendrá el relato de seis mujeres que han vivido violencia y que tienen un animal de compañía, con ello se podrá conocer las bondades y los retos de este tipo de intervención.

Sin duda este tipo de modelos de intervención en violencia contra las mujeres hace referencia a la terapia de orientación feminista, que tienen como base los trabajos de la llamada Survivor Therapy (Walker,1994), enfocada al empoderamiento y superación del trauma apartándose de los modelos tradicionales de atención. Estas intervenciones de orientación feminista se enfocan entre otros a que las mujeres que han vivido violencia 
puedan tomar de conciencia y comprensión de lo vivido, focalizándose en trabajar en la recuperación de los déficits psicológicos como autoestima, autonomía e independencia, reconstruyendo lo personal, lo social, lograr el empoderamiento de las mujeres hacia una nueva vida (Bosch, Ferrer, Alzamora, 2005).

Existen también programas altamente estructurados de empoderamiento basados en la terapia feminista como es el caso del programa STEP de 12 pasos, diseñado para abordar y disminuir los síntomas de ansiedad, afectos negativos asociados al trauma y la violencia de género apoyando la regulación emocional, agresión, competencia social y relaciones interpersonales (Jungersen,2017). Este tipo de terapia de orientación feminista se pondera el ecofeminismo que implica el retorno a la naturaleza, donde sin duda esta la relación humano-animal (Guerrero, 2010).

La inclusión de animales no es una panacea, un sustituto a terapias o tratamientos, es un complemento y tiene sus requisitos y diferencias como lo es la terapia asistida con animales o TAA y la actividad asistida por animales o AAA, la TAA, es una intervención dirigida a un objetivo en la que el animal cumple con criterios específicos y es una parte integral del proceso de tratamiento que es dirigido por un profesional de la salud, con experiencia o con una especialización y dentro de su profesión, incluye metas y objetivos específicos y medición del progreso, mientras que la AAA se da en otros entornos como el educativo, visitas hospitalarias, en asociación con animales que cumplan criterios específicos, se diferencia con la TAA en que hay ausencia de tratamiento específico, metas y los voluntarios o llamados proveedores de tratamiento no están obligados a tomar notas o registros detallados. (Delta Society,1996), (Marino, 2012), (Cabra,2012).

Además de las anteriores, hay otras formas mas precisas para que los animales puedan constituirse en camino para el cambio terapéutico. (Serpell, 1981, citado en Abellán, 2008) son el camino instrumental, pasivo y antropomórfico; el instrumental se refiere a perros guía, caballos especializados, perros terapeutas, debidamente capacitados para ello incluyendo su certificado, el pasivo se refiere a la interacción a partir de la observación y contemplación de animales, y el antropomórfico se refiere específicamente a los animales de compañía con capacidad de formar un vínculo afectivo con el dueño, por lo que esta última intervención beneficia a las personas al realizar un vínculo se sienten no amados, rechazados, dañados o socialmente solos.

Se conocen beneficios físicos, psicológicos y sociales de la interacción con animales (Gutiérrez, Granados, Piar, 2007),(Jofré, 2005), (San Joaquín, 2002), (Jiménez, et al.,2012) en términos de promover la actividad física, centrar la atención, estimulación táctil, mejorar destrezas motoras finas, crear placer y disminuir el dolor, generar afecto incondicional, aumentar la interacción social y catalizador social al establecer lazos con el exterior, propicia la intimidad y la capacidad de comunicación verbal y no verbal, mejora el sentido del humor, aumento de la autoestima, favorece el contacto físico, la seguridad, propicia el juego y con ello la constancia, genera demostraciones de afecto, desarrolla la afinidad y es un protector de las personas contra la soledad, depresión.

Sin duda los perros hacen un papel de apoyo, cercanía, ayuda, sanación, cura, propician la cercanía, las caricias, permiten volver a tocar y ser tocado mitigando el dolor, heri- 
das y contracturas emocionales, su presencia ayuda al restablecer el vínculo de confianza en sí mismo y en el otro, permitiendo la cercanía, y la creación de lazos emocionales, además de cuidado recíproco, al respecto se exponen las propiedades mediadoras de los animales de compañía al establecer el vínculo humano-animal, en tres áreas principales, un efecto lubricante social el cual se refiere a la supuesta capacidad de las mascotas para catalizar las relaciones sociales, la capacidad de las mascotas para servir como una especie de vínculo moral con otras categorías de animales y finalmente el animal dentro, lo que sugiere que los animales de compañía pueden ayudar a conectar o reunir a las personas con algo fundamental dentro de sí mismas (Serpell,2000).

\section{Método}

Se trata de un estudio exploratorio con enfoque cualitativo, según (Sampieri, 2018) los estudios exploratorios tienen como propósito familiarizarnos con un planteamiento, desconocido o novedoso, sentando las bases para estudios mas profundos, las metodologías cualitativas se orientan hacia la comprensión de las situaciones únicas y particulares, se centran en la búsqueda de significado y de sentido que les conceden a los hechos los propios agentes, en cómo viven y experimentan ciertos fenómenos o experiencias los individuos o los grupos sociales a los que investigamos y es un método que permita dar cuenta de este problema desde la perspectiva de los actores sociales involucrados (Gómez, Roquet, 2012).

Se utilizará el relato de vida tomando en cuenta estos, no son ni la vida misma, ni la historia misma, sino una reconstrucción realizada en el momento preciso de la narración y en la relación específica con un narratario, por lo que es mas breve y preciso (Cornejo,Mendoza, Rojas, 2008).

\section{Participantes}

La muestra elegida por conveniencia es de 6 mujeres de edades entre 30 y 60 años, las cuales comparten como característica haber vivido violencia por parte de su pareja hombre y tener al menos un animal de compañía, todas las mujeres ya no se encuentran en la relación violenta, la descripción de las participantes es la siguiente y se anexa la fecha en la que fueron entrevistadas. Para fines de confidencialidad y anonimato no se conservaron los nombres de los entrevistados; en cambio, se les asignó un seudónimo que las representara.

$\begin{array}{ll}\text { Leo } & \text { (60 años, relato, enero 2019) } \\ \text { Carmen } & \text { (45 años, relato, marzo 2019) } \\ \text { Gaby } & \text { (35 años, relato, abril 2019) } \\ \text { Loly } & \text { (30 años, relato, mayo 2019) } \\ \text { Clau } & \text { (32 años, relato mayo 2019) } \\ \text { Maria } & \text { (38 años, relato, junio 2019) }\end{array}$




\section{Técnicas}

Se utiliza la entrevista enfocada a conocer el relato respecto al apoyo o papel de su perro en su recuperación, por lo que la pregunta se genera con base al marco teórico revisado y se realiza de manera semiestructurada para conocer: ¿De qué manera ha apoyado su animal perro en el proceso recuperación luego de haber vivido violencia contra las mujeres?

\section{Procedimiento}

Las mujeres fueron referidas por el centro de salud al que acuden a su proceso terapéutico por la violencia vivida, mismo centro que conoce de manera formal la intensión del estudio acerca de recabar estos relatos respecto a la violencia contra la mujer y la presencia de su perro, por lo que al detectar durante su proceso que las mujeres que habían vivido violencia tenían un perro fueron oportunamente invitadas y remitidas para colaborar con el estudio, luego de aceptar, se procedió a llevar a cabo la entrevista con cada mujer misma que se realizó en el centro de salud ya conocido por ellas, se procedió a recabar los relatos en una sola sesión de 50 minutos, en los meses de enero a junio del 2019 hasta obtener los seis casos, el relato se dio de manera voluntaria previo a explicarles el objetivo del estudio y obtener su consentimiento informado y permiso para la toma de notas.

Después de obtener los relatos se procedió al análisis de datos y codificación de la información siguiendo los principios de la teoría fundamentada (Glaser y Strauss, 1967), que implica identificar categorías teóricas derivadas de los datos en los relatos, generando así un método comparativo constante entre los relatos, ello implica que el investigador compare estos con los conocimientos teóricos fundados lo cual permite identificar las diferencias y similitudes de los datos, derivándose así las categorías teóricas que permitan comprender el objetivo del estudio.

La Teoría Fundamentada o TF busca conocer cómo las personas interpretan su realidad, es un proceso interpretativo que depende de la sensibilidad del investigador a los elementos tácitos de los datos o a los significados y las connotaciones que pueden ser aparentes como resultado de una lectura superficial de contenidos reveladores (Páramo, 2015).

\section{Resultados}

Seguidamente de tener las entrevistas y conocer los relatos, se procede a generar las dimensiones en las cuales se aprecia como se pondera el vínculo humano animal, se describe como los animales actúan como soporte emocional en sus encrucijadas emocionales y como potencializan la fuerza de ellas mismas y en si son catalizadores de cambio en sus estados emocionales pudiendo modificar estos, clasificándose los hallazgos de la siguiente manera: 


\subsection{Vínculo Humano-Animal}

Se aprecia en los relatos como la relación que se genera es estrecha, genuina, de afecto, que podría considerase exclusiva, en sí el vínculo que se genera es único y simétrico, un vínculo interespecies, en este vínculo se identifica cuatro principios básicos de la interacción hombre-animal que permiten el establecimiento del vínculo: seguridad, intimidad, afinidad y constancia. La seguridad se refiere a que la presencia del animal salvaguarda y protege a los humanos y tiene un efecto tranquilizador mediante acciones de los animales y mejora la percepción de las relaciones sociales al ser mediador en su vida social, la intimidad entre dueño y mascota ocurre de manera espontánea, el dueño habla y acaricia a su mascota, con un lenguaje corporal relajado que se da sólo cuando aquel lo quiera y necesite, ya que su mascota siempre está dispuesta a jugar y a ser acariciada, este efecto de intimidad se ve fuertemente soportado en la capacidad de comunicación entre humanos y animales quienes tienen habilidad especial para comprender señales verbales y no verbales de los humanos, reforzando la intimidad y constancia al grado de establecer relaciones de parentesco, los beneficios que proveen las mascotas son perdurables en el tiempo y recordados incluso si la mascota ya no esta por alguna razón. (Katcher,1993, citado por Gutiérrez, Granados, Piar, 2007).

"Son como mis hijos, son siete y creo que cada uno de ellos lo fui adoptando ya que al ver sus ojos me veía yo en ellos y no los podía abandonar a como a mi me lo hicieron, uno de ellos enfermó de cáncer y falleció, ese me ayudaba a levantarme de la cama desde temprano, para mi que se comió mi depresión, era un perro que si yo no me despertaba o no me quería levantar hacia ruido, ladraba, o se echaba encima de mí, pero me levantaba". (Leo)

"Tenía gran apego con ella, su nombre era Amor, así le puse para tener a quien decirle mi amor, estaba sola y en la familia de mi exmarido no la querían allí y a mí tampoco, así que cuando me fui me la llevé, ella esperaba pacientemente a que yo regresara de trabajar y desde lejos le gritaba, mi amor, y ella me ladraba, tuvo cachorros y me quede con uno, se llama cachito y ahora es quien me acompaña, amor murió por una infección terrible, ahora yo le cuento a cachito de su mamá y de cómo ambas salimos de ese lugar, mi amor vive dentro de mí, ella me regresó el amor propio". (Carmen)

"Al principio no soportaba su presencia, era de mi exesposo y lo abandono al igual que a nosotros, yo cuando lo veía, le decía que se fuera, después poco a poco, pasando los días y muy quietecito, muy poco a poquito se acercaba a mi sin que me diera cuenta, con mucho respeto, no me gustaba verlo a la cara ya que tiene un ojo de un color y otro de otro, de verdad que lo rechazaba, sin embargo su cercanía, contacto poco a poco, me fueron haciendo que lo quisiera, a veces hasta le reclamaba lo que me había hecho su ex dueño, pero él supo estar, a tal grado que hoy lo cuido mucho y le agradezco que estuvo paciente, constante y leal, ya le pregunte por que de sus ojos y entendí que todo depende de cómo se miren las cosas, lo amo, se llama Royal." (Clau)

Se aprecia como los animales de compañía, las apoyan a generar vínculos afectivos mas positivos, donde incluso se reflejan a si mismas y a su problemática, pueden ver 
afuera lo que no se aprecia o cuesta trabajo ver internamente, sin duda los vínculos sanos apoyan a reparar otros vínculos no positivos o destructivos, se considera concretamente en mujeres que han vivido violencia la capacidad para conceptualizar otros vínculos sanos y estilos de apego diferentes a los que alguna vez vivieron en la violencia y como posiblemente este vínculo positivo ayude a disolver vínculos nocivos, de allí la importancia de este nuevo vínculo. (Loubat, Ponce, Salas, 2007)

\subsection{Encrucijada de emociones}

Las emociones tienen un papel muy específico en el ser humano en varios sentidos, sirven como un lenguaje para comunicarnos entre los seres humanos y con los animales, las emociones desempeñan un papel mecanismo de supervivencia e indican cuando protegerse, pero, también pueden perder ese valor de adaptación y entonces se vuelven perjudiciales para la salud física y mental, la curación emocional lleva su tiempo hasta recorrer el camino hacia el equilibrio emocional y con ello salir así del punto central de la encrucijada donde se precisamente se cruzan los caminos emocionales que en ocasiones refiere son desequilibrantes, el objetivo sera entonces integrar al ser humano en lo que piensa, siente y hace. (Bizkarra,2005)

En los relatos se muestra como los animales de compañía, ayudan a sobrepasar y resolver cada encrucijada emocional y con ello no permanecer en la emoción negativa que genera síntomas y signos no deseables.

"Son dos, antes tenía otra y se murió por estar tanto tiempo sola en ocasiones pienso que debí haberle dado a la otra pobre que se me murió, una amiguita con quien jugar pero ya ni modo, luego de haber adoptado a estos dos se llaman los niños, me doy cuenta de la importancia de estar bien acompañada, cuando me pongo triste ellos me acarician con sus bigotes, me miran fijamente hasta me hacen volver a sonreír y entonces me dan una lamida en la barbilla y siguen jugando, actualmente soy voluntaria en un refugio..." (Gaby)

"Lloran cuando lloro, se angustian cuando estoy angustiada, pero siempre con un ladrido rompen ese abismo negro y no me dejan caer..." (Leo)

"Los perros apoyan incondicionalmente a los humanos y ellos detectan cuando estas deprimida, al acariciarlos y saber que están allí, sin ningún interés, te ayudan anímicamente a sentir puro amor, el recibir su cariño te hace sentir menos mal cuando tienes problemas, el acariciarlos es terapéutico..." (María)

"Royal, también cuida de mis hijos y cuando los regaño, me ladra, muy fuerte y se pone entre ellos y yo, eso era antes cuando les gritaba o pegaba cuando no me controlaba, él no me dejaba pasar defendía a mis hijos y su ladrido me ayudaba a salir de ese enojo a reaccionar y calmarme..." (Clau)

Se logra observar como las animales de compañía les han apoyado incondicionalmente, no solo mueven las emociones de esta confluencia de emociones y a pasar tiempos y estados emocionales difíciles y a auto controlarse, sino que además han despertado sen- 
timientos como amor, lealtad, fidelidad, amistad, entre otros, al respecto apuntan que se han encontrado diferencias entre hombres y mujeres frente a la función que representa poseer una mascota; mientras que las mujeres reportan una función de facilitador social, incluyendo las mascotas como ayuda para superar tiempos difíciles, los hombres consideran razones prácticas tales como que las mascotas facilitan el ejercicio o sirven alguna función útil (Staats, Pierfelice, 2006, citados en Gutiérrez, Granados, Piar, 2007).

\subsection{Restauradores de fuerza y energía}

Sin duda los animales de compañía son catalizadores para potencializar y acelerar la recuperación de emociones, es importante mencionar que la violencia que vivieron mermo su energía, su voluntad, al grado del sometimiento, por ello los animales de compañía son parte de la red de apoyo que sin duda apoyarán a movilizar y activar a la persona a mantenerse en movimiento, se conoce que la participación de animales apoya a la motivación al ser un elemento novedoso, divertido, dinamizador ya que desde el punto de vista psicológico los perros adoptan una actitud afectiva y de gran apego hacia el ser humano, sin hacer juicios de valor, además de que pueden ser excelentes guías, son lúdicos, afectivos, juguetones y están a su lado fielmente durante toda su vida, señalando que acariciar a un perro regula los valores de tensión arterial, frecuencia de respiración y latidos cardíacos, entre otros. (Oropesa, et al., 2009)

“Ellos saben lo que me pasa, y sanan la violencia que viví, con una sacudidita de su cuerpecito liberan energía que no quieren y así mismo se va la mía con una sacudidita, yo también aprendí a sacudirme como ellos para que se vayan las ideas y los recuerdos." (Leo)

"Se llama Aquiles, por que solo tiene una debilidad, actualmente esta muy enfermo, él ya me trasmitió toda su energía a mi cuerpo y a mi corazón, ahora yo lo asisto a él por que apenas puede pararse, se para y se vuelve a caer, pero que crees, no se rinde, nunca lo hace y se vuelve a levantar, como si dijera de nuevo vas para arriba..." (Loly)

“...por que ellos reciben tu energía y la cambian en positivo, es como un paliativo, son excesivamente sensibles, no solo cuando hay un maltrato o violencia, con la depresión en general te ayudan muchísimo, al igual que el ronroneo de los gatos, el ronroneo te relaja son vibraciones continuas que provocan que te apacigües y vuelvas a retomar vuelo." (María)

"Esa violencia desapareció, ambos son mis caminos de luz, son como mis hijos, los reales ahora están lejos y en sus vidas, pero estos dos, son mis caminos de luz, son seres divinos eso creo yo, son transmutadores y limpiadores del alma, soy feliz con ellos y también con los del albergue, por eso estoy allí para que cada persona que lo requiera se lleve a uno a casa, les cambia la vida, les de energía y ellos también se la cambian al perrito." (Gaby)

Valga la siguiente reflexión para terminar de exponer como los animales de compañía revitalizar, dan fuerza y organizan el caos, se menciona que todos somos seres latientes, ya que lo primero que percibimos y más nos tranquiliza es el latido del corazón materno, el latido nos hace saber que no estamos solos en la vida, la especie humana tenemos 
la capacidad de sentir ese vacío existencial, difícil tanto de explicar como de entender, la medicina para el alma humana se encuentra en la naturaleza (San Joaquín, 2002).

Por eso cuando se reúnen el latido humano y el canino forman una sinergia de amor, apego, compañía, sanación, curación, cuidado y con ello la restauración de la fuerza y de la energía, todo vuelve a latir.

\section{Discusión}

Los resultados en términos de la importancia del vinculo humano- animal, la encrucijada de emociones y restauradores de fuerza y energía permiten constatar que los perros apoyan a depurar la violencia y a restaurar el equilibrio, se aprecia entre otros que el vínculo que se genera con el perro es estrecho, genuino, único y simétrico cubriendo los elementos básicos del vínculo interespecies: seguridad, intimidad, afinidad y constancia.

Se aprecia que el vínculo le ayuda a las mujeres a reflejar su propia problemática en el perro, lo cual les apoya a poner afuera lo que no pueden ver o manejar dentro de sí mismas y les apoya a identificar también vínculos no positivos.

Falta tomar en cuenta que los perros también han vivido la violencia, el rechazo, que también fueron abandonados o dañados por sus dueños y que de alguna manera se adaptaron a su nuevo cuidador.

Respecto a la encrucijada de emociones se constata que en momentos de desequilibrio el perro se mimetiza al copiar la emoción para que la persona la pueda apreciar en el exterior, resulta por demás interesante cómo se rompe la encrucijada emocional con un ladrido, sacudida, lamida, contacto visual, caricias, en si una alarma que le apoya a la mujer a salir del estado emocional negativo, regresar a su equilibrio, apreciándose como los perros pueden ayudar a conectar o reunir a las personas con algo fundamental dentro de sí mismas, a partir de recibir del perro el cuidado, alerta y regulación, cualidades que por la violencia se menguaron.

La interacción y el vínculo ha despertado en ellas acciones prosociales tal como se describe de lubricante social en las relaciones con otros tales como ser voluntarias en refugios caninos o tener la disposición de hablar de si mismas para apoyar a otros.

Se aprecia que los perros permiten restaurar la fuerza, energía rítmica y reguladora al activar la capacidad correguladora de cuidado recíproco, la actividad somato sensorial repetitiva y rítmica al acariciarlos y la cercanía les permite restablecer una relación de confianza.

Se identifican las siguientes áreas de oportunidad, este estudio es solo a partir de analizar la inclusión de animales de compañía como parte de la red de apoyo con la que cuentan las mujeres, sin embargo, aún dista de ser una TAA o AAA ya que el canino no esta adiestrado para ello, sin embargo, si a pesar de la falta de adiestramiento hacen el papel tan importante en el apoyo con una simple inclusión, si elabora una TAA seguro tendrá éxito, es decir, el visibilizar la importancia de su inclusión con seguridad apoyará a adiestrar a caninos para la recuperación en casos de violencia no solo contra la mujer, sino de 
cualquier tipo, es decir esta investigación sienta las bases para poder crear protocolos de atención y rehabilitación psicosocial.

Trabajar con animales de apoyo en este caso caninos requiere de un trabajo largo y constante que también incluye apego al manejo ético y responsabilidad por parte del ser humano, por lo que esta investigación pretende sentar bases para la utilización de este tipo de intervenciones que nos invitan al retorno con nuestro propio animal interno y nos invita a reparar vínculos rotos por el hombre pero reparados por los animales.

\section{Referencias}

AMNISTÍA INTERNACIONAL (2001) Cuerpos rotos, mentes destrozadas: tortura y malos tratos a mujeres. Madrid, 2001 p.4.

ARCE, R. (2014). El sistema de Evaluación Global en casos de violencia de género: Huella psíquica y testimonio. Jornadas de Psicología, 99(4), 26-27.

ABELLÁN, R. M. (2008). La terapia asistida por animales: una nueva perspectiva y línea de investigación en la atención a la diversidad. Indivisa. Boletín de estudios e investigación, (9), 117-143. p. 130.

BIZKARRA, K. (2005). Encrucijada emocional. Bilbao: Desclée de Brouwer.

BOSCH Fiol, E., Ferrer Pérez, V. A., \& Alzamora Mir, A. (2005). Algunas claves para una psicoterapia de orientación feminista en mujeres que han padecido violencia de género. Feminismo/s. N. 6 (dic. 2005); pp. 121-136.

BOURDIEU, P. (2012). Symbolic violence. Revista latina de sociología, (2), 1-4.

CABRA Martínez, C. A. (2012). Terapia asistida con animales. Efectos positivos en la salud humana.

CASTELLANOS, V. (2017). Violencia contra la mujer a la luz de los Derechos Humanos: Resignificación de la vivencia.(Tesis Doctoral). Universidad Juárez Autónoma de Tabasco.

CONFERENCIA MUNDIAL SOBRE LAS MUJERES IV (1995) Beijing, China.

CORNEJO, M., Mendoza, F., \& Rojas, R. C. (2008). La investigación con relatos de vida: pistas y opciones del diseño metodológico. Psykhe (Santiago), 17(1), 29-39.

DELTA SOCIETY. (1996). Standards of practice for animal-assisted activities and therapy. Bellevue, WA: Author.

FERNÁNDEZ, O., del Prado, M., \& González Sánchez, P. (2012). Las víctimas invisibles de la Violencia de Género. Revista clínica de medicina de familia, 5(1), 30-36.

GARCÍA-MORENO, C. "Violencia contra la mujer." Género y equidad en la salud 2000. p 12. FLYNN, K. (2018). Animal Assisted Therapy for Adult Domestic Violence Survivors. Thinking Matters Symposium. 154.

GLASER, B. \& Strauss, A. (1967). The discovery of grounded theory. Chicago: Aldine Press. GÓMEZ, S., \& Roquet, J. V. (2012). Metodología de la investigación. México: Red tercer milenio. p. 47.

GUERRERO, O. F. (2010). Cuerpo, espacio y libertad en el ecofeminismo. Nómadas. Critical Journal of Social and Juridical Sciences, 27(3). 
GUTIÉRREZ, G., Granados, D. R., \& Piar, N. (2007). Interacciones humano animal: características e implicaciones para el bienestar de los humanos. Revista colombiana de psicología, 16(1), 163-184.

HAMAMA, L., Hamama-Raz, Y., Dagan, K., Greenfeld, H., Rubinstein, C., \& Ben-Ezra, M. (2011). A preliminary study of group intervention along with basic canine training among traumatized teenagers: A 3-month longitudinal study. Children and Youth Services Review, 33(10), 1975-1980.

HIDALGO-Ruzzante, N., Gómez, P., Bueso-Izquierdo, N., Jiménez, P., Martín Del Moral, E., \& Pérez-García, M. (2012). Secuelas cognitivas en mujeres víctimas de violencia de género. In Congreso para el estudio de la violencia contra las mujeres.

JIMÉNEZ, X.O., Hernández, R. L., \& Ramírez, M. T. G. (2012). Terapia asistida por perros en el tratamiento del manejo de las emociones en adolescentes. Summa Psicológica UST, 9(2), 25-33.

JOFRÉ, M. (2005). Visita terapéutica de mascotas en hospitales. Revista chilena de infectología, 22(3), 257-263.

JUNGERSEN, TS (2017). El Programa de Empoderamiento de Terapia de Sobrevivientes para la Violencia de Género.

KRUG, E. G., Dahlberg, L. L., Mercy, J. A., Zwi, A. B., \& Lozano, R. (2003). Violencia, un problema mundial de salud pública. In Informe mundial sobre la violencia y la salud (Vol. 588, pp. 3-23). OPS.

LAGARDE, M. (1996). Identidad de género y derechos humanos la construcción de las humanas Guzmán Stein, Laura y Silvia Pacheco (comps.) Estudios básicos de derechos humanos IV. Instituto Interamericano de Derechos Humanos, San José, Costa Rica. 1996, pp.1-32.

LAMMOGLIA, E. (2009) Violencia Emocional. México: Grijalbo.

LIN CHING, R. (2003). Propuesta de valoración del daño psicológico en materia de violencia doméstica. Medicina Legal de Costa Rica, 20(2), 53-67.

LOUBAT, M., Ponce, P., \& Salas, P. (2007). Estilo de Apego en Mujeres y su Relación con el Fenómeno del Maltrato Conyugal. Terapia psicológica, 25(2), 113-122.

MARINO, L. (2012). Construct Validity of AnimalAssisted Therapy and Activities: How Important Is the Animal in AAT?. Anthrozoos: A Multidisciplinary Journal of The Interactions of People \& Animals, 25(Supplement 1), 139-151.

MUÑOZ-López, L. I. (2017). Terapias asistidas con animales. Universidad de Jaen (tesis de maestria) Andalucía.

PÁRAMO Morales, D. (2015). La teoría fundamentada (Grounded Theory), metodología cualitativa de investigación científica. Pensamiento \& gestión, (39), 1-7. OMS Violencia contra la mujer. Violencia de pareja y violencia sexual contra la mujer. Nota descriptiva $\mathrm{N}^{\circ}$ 239. Noviembre del 2017. http://www.who.int/mediacentre/factsheets/fs239/es/.

OMS (2013) Departamento de Salud Reproductiva e Investigación,Escuela de Higiene y Medicina Tropical de Londres, Consejo Sudafricano de Investigaciones Médicas (2013). Estimaciones mundiales y regionales de la violencia contra la mujer: prevalencia y efectos de la violencia conyugal y de la violencia sexual no conyugal en la salud 
OMS (2013). Comprender y abordar la violencia contra las mujeres abordar. https://apps. who.int/iris/bitstream/handle/10665/98862/WHO_RHR_12.43_.

ONU MUJERES (2018) Hechos y cifras: Acabar con la violencia contra mujeres y niñas. Diferentes formas de violencia http://www.unwomen.org/es/what-we-do/endingviolence-against-women/facts-and-figures\#notes.

ONU Mujeres, a partir de INEGI, Estadísticas vitales de mortalidad, CONAPO, Conciliación de la Población de México 1970-2015 y proyecciones de la población de México 2016-2050 (2016-2017).

ONU Organización de las Naciones Unidas (2019). Día Internacional de la Mujer, 8 de Marzo.http://www.un.org/es/events/womensday/.

OPS (2003) Publicación Científico Técnica No. 588. Washington DC, 2003.p.11

OROPESA Roblejo, P., García Wilson, I., Puente Saní, V., \& Matute Gaínza, Y. (2009). Terapia asistida con animales como fuente de recurso en el tratamiento rehabilitador. Medisan, 13(6), 0-0.

RIVERO, Alejandro (2014). La violencia contra las mujeres una forma de tortura. Instituto Nacional de las Mujeres. Comunicado de prensa No1. -8 de Enero de 2014. inmujeres. gob.mx .

SAMPIERI, R. H. (2018). Metodología de la investigación: las rutas cuantitativa, cualitativa y mixta. McGraw Hill. México.

SAN JOAQUÍN, M. Z. (2002). Terapia asistida por animales de compañía. Bienestar para el ser humano. Temas de hoy, 143-149.

SERPELL, J. A. (2000). Creatures of the unconscious: Companion animals as mediators.

SESSAREGO, C. F. (2003). Deslinde Conceptual entre" Daño a la Persona", "Daño al Provecto de Vida" y "Daño Moral". Foro Jurídico, (02), p.27.

SORIANO Izquierdo, M. (2018). Prevención de la violencia de género durante la adolescencia a través de la Hipoterapia.

SUÁREZ, M. G. (2019). Uso y abuso de los animales: responsabilidades éticas de la Psicología. Wímblu, 14(2), 59-82.

TESESCHI, Philip and Jenkins, Molly Anne, "Transforming Trauma: Resilience and Healing Through Our Connections With Animals" (2019). Purdue University Press Book Previews. 31.

WALKER, L. E. A. (1994). Abused women and survivor therapy: A practical guide for the psychotherapist. Washington, DC, US: American Psychological Association. 